\title{
Voltage-Driven DNA Translocations through a Nanopore
}

\section{Citation}

Meller, Amit, Lucas Nivon, and Daniel Branton. 2001. Voltage-driven DNA translocations through a nanopore. Physical Review Letters 86(15): 3435-3438.

\section{Published Version}

http://dx.doi.org/10.1103/PhysRevLett.86.3435

\section{Permanent link}

http://nrs.harvard.edu/urn-3:HUL.InstRepos:3109361

\section{Terms of Use}

This article was downloaded from Harvard University's DASH repository, and is made available under the terms and conditions applicable to Other Posted Material, as set forth at http:// nrs.harvard.edu/urn-3:HUL.InstRepos:dash.current.terms-of-use\#LAA

\section{Share Your Story}

The Harvard community has made this article openly available.

Please share how this access benefits you. Submit a story.

Accessibility 


\title{
Voltage-Driven DNA Translocations through a Nanopore
}

\author{
Amit Meller, ${ }^{1,2, *}$ Lucas Nivon, ${ }^{1,3}$ and Daniel Branton ${ }^{3}$ \\ ${ }^{1}$ The Rowland Institute for Science, Cambridge, Massachusetts 02142 \\ ${ }^{2}$ Department for Biomedical Engineering, Boston University, Boston, Massachusetts 02215 \\ ${ }^{3}$ Department of Molecular \& Cellular Biology, Harvard University, Cambridge, Massachusetts 02138
}

(Received 8 December 2000)

\begin{abstract}
We measure current blockade and time distributions for single-stranded DNA polymers during voltagedriven translocations through a single $\alpha$-hemolysin pore. We use these data to determine the velocity of the polymers in the pore. Our measurements imply that, while polymers longer than the pore are translocated at a constant speed, the velocity of shorter polymers increases with decreasing length. This velocity is nonlinear with the applied field. Based on this data, we estimate the effective diffusion coefficient and the energy penalty for extending a molecule into the pore.
\end{abstract}

DOI: $10.1103 /$ PhysRevLett.86.3435

The translocation of biopolymers such as RNA, DNA, and polypeptides across membranes is ubiquitous in biology. Examples include the movement of RNA molecules and transcription factors across nuclear pores [1], phase infection [2], the uptake of oligonucleotides by specific membrane proteins [3], and the incorporation of proteins into specific membranes in nearly all cells [4]. Alphahemolysin $(\alpha-\mathrm{HL})$, the proteinaceous membrane channel produced by $S$. aureus, can serve as a model system to study the physics of this translocation process. $\alpha$-HL can self-assemble into a lipid bilayer, in effect creating an aqueous nanopore (inside diameter $\sim 2 \mathrm{~nm}$ ) across the lipid membrane. In vitro experiments show that single-stranded DNA polymers can be driven through this nanopore by an external electric field [5]. We reconstituted $\alpha$-HL in a model planar bilayer separating two small compartments and applied a fixed voltage bias across the membrane to generate a steady electrolyte current through the pore. Single polymer translocations were observed by recording blockage of the ionic current as polynucleotides moved through the pore [5]. More recently, it has been shown that careful characterization of the ionic current signals allows real-time discrimination between different types of short homopolymers of RNA [6] and DNA [7] molecules. Although these findings suggest that such a system could be developed into an ultrafast method of DNA sequencing, it is necessary first to elucidate the physical mechanism underlying polymer translocation through biological pores.

Recent theoretical analyses have investigated the entry and subsequent translocation of polymers through nanopores [8-10]. Sung et al. developed a statistical model describing the polymer translocation as a diffusion process across a free energy barrier generated by the reduced entropy of free polymers (before and after translocation) as compared to "anchored" polymers (during translocation) [8]. Muthukumar employed a similar model but adjusted it to the case where the polymer's translocation rate is constant [9]. Lubensky et al. considered polymers that were driven electrophoretically through the pore and assumed to have strong interactions with the pore walls
PACS numbers: $87.14 . \mathrm{Gg}, 87.15 . \mathrm{Tt}$

[10]. The driving force was approximated as $F \approx z e V / a$, where $e z$ is the effective charge per base, $e$ is an elementary unit charge, $V$ is the electrostatic potential drop through the pore, and $a$ is the base to base distance along the DNA polymer. Assuming $z=1, V=125 \mathrm{mV}$, and $a=4 \AA$, the force is estimated to be $F \approx 5 k_{B} T / a \approx$ 44 piconewtons (pN). Equivalently, $F$ can be seen as determining the ratio of the probabilities of moving one unit forward and one unit backward, or $e^{F a / k_{B} T}$, averaged over all the monomers. On a macroscopic level, the process can be modeled by a one-dimensional diffusion equation with an average drift velocity, $\nu$, and an effective diffusion coefficient, $D_{\text {eff }}$, that takes into account all possible interactions, including friction [10].

Here, we present a new set of experimental results that sheds light on the physics governing the driven motion of DNA through narrow pores. By measuring the translocation velocity of single stranded DNA (ssDNA) polymers as a function of their length and the driving force, we show two distinct regimes, one for translocations of short polymers, the other for long polymers. These regimes are separated by a sharp transition, which is set by a length scale corresponding to the pore length, $d$. The translocation velocity for polymers shorter than $d$ depends strongly on length, whereas this velocity is independent of length for polymers longer than $d$. This behavior is not accounted for by the existing models, since they either assume a zero membrane thickness $(d=0)$ or assume very long polymers. We also find that the velocity has a nonlinear dependence on the applied electric field. From these measurements, we estimate the driving force and the effective diffusion coefficient, both of which are significantly less than prior estimates [10].

A single protein nanopore ( $\alpha$-HL from Staphylococcus aureus) was self-assembled in a phospholipid bilayer membrane (diphytanoyl phosphatidylcholine) supported horizontally on a $25 \mu \mathrm{m}$ aperture fabricated at one end of a small Teflon tube, as previously described [7]. The membrane separated two Teflon chambers, each containing $1 \mathrm{M} \mathrm{KCl}, 1 \mathrm{mM}$ Tris-EDTA buffer $(p \mathrm{H} \mathrm{8.5)}$ 
in contact with a $\mathrm{Ag}-\mathrm{AgCl}$ electrode [Fig. 1(a)]. The entire apparatus was embedded in a copper enclosure so that the temperature of all the solutions and components could be regulated $\left( \pm 0.05^{\circ} \mathrm{C}\right)$. Single-stranded DNA (Midland Certified Reagents, Midland, Texas) below 40 bases was reverse phase grade and used without further purification. Longer polymers (60-100 bases) were size purified in-house. DNA was injected on the negative side [see Fig. 1(a)] and a voltage of $70-300 \mathrm{mV}$ was applied across the pore using a commercial head-stage [7].

Figure 1(b) is a typical current trace showing three translocating DNA molecules at $2{ }^{\circ} \mathrm{C}$ and $120 \mathrm{mV}$. As each molecule enters and translocates through the pore, the current drops from its initial open pore value $(\sim 70 \mathrm{pA})$ to its blocked level $(\sim 5 \mathrm{pA})$. As the DNA molecule clears the pore, the open pore current level is restored. Between 500 and 1500 translocation events were recorded separately for each type of DNA, and each voltage setting. Translocation events were defined as those that decreased the average current to less than $35 \%$ of the open pore value [see Fig. 1(b)]. We characterize each translocation event by its average normalized blocked current $I_{B}=\left\langle I_{\text {blocked }}\right\rangle /\left\langle I_{\text {open }}\right\rangle$ and by its translocation duration $t_{D}$.

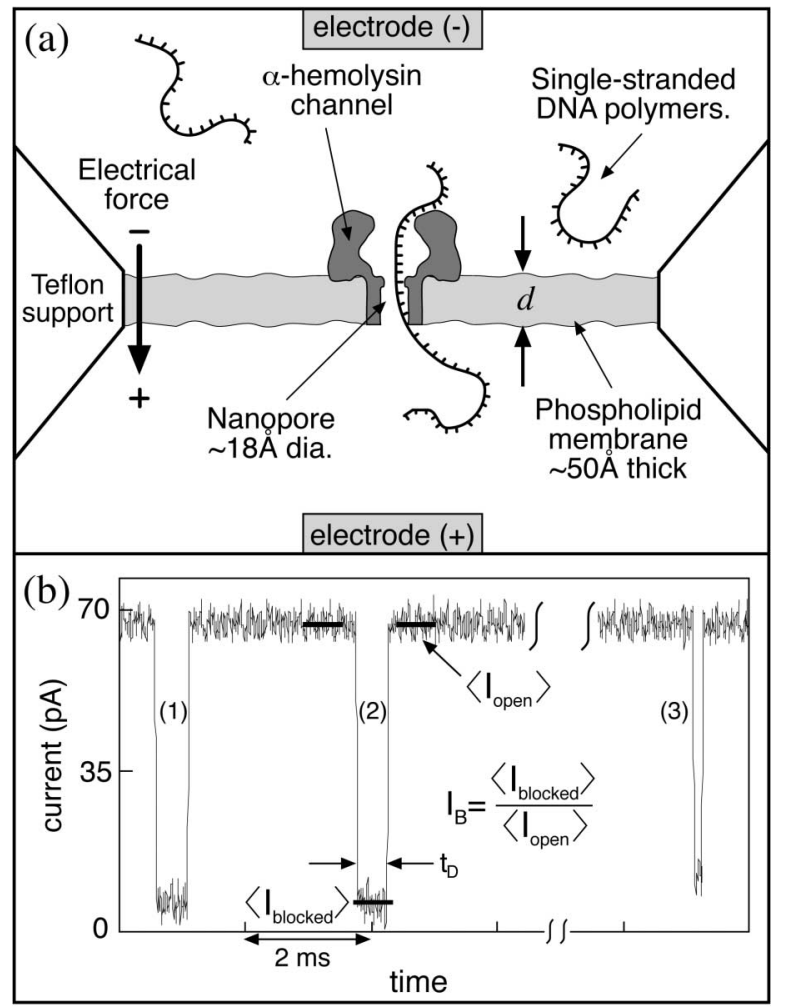

FIG. 1. (a) Single-stranded DNA molecules (negatively charged) and salt ions are electrically driven through a single $\alpha$-hemolysin protein pore embedded in a phospholipid membrane. Most of the ionic current through the pore is blocked during DNA passage. (b) Three representative translocation current blockades are shown for 15 mer ("1" and "2") and for 7mer ("3") poly(dA). For each event we measured the translocation time, $t_{D}$, and the average event blockade, $\left\langle I_{B}\right\rangle$.
The single molecule nature of our data allows us to perform a detailed statistical analysis of the DNA sliding process in the $\alpha$-HL pore. For all the polymer types tested, the histogram of current blockades exhibited a Gaussian distribution of values (Fig. 2, inset), whose peak we define as $I_{P}$. If we interpret $I_{P}$ as a measure of the fraction of the pore volume from which ions are excluded by the translocating polymers, it follows that, for polymers shorter than the pore length (or $L \leq d$ ), the $I_{P}$ values will vary with $L$, while for long polymers $(L>d)$ they will be $L$ independent. $L$ is the polymer's contour length, and $L=N a$, where $N$ is the number of monomers. Figure 2 shows representative $I_{P}$ data for poly $(\mathrm{dA})$ (polydeoxyadenylic acid) in the length range 4-100 bases. As expected, $I_{P}$ depends very weakly on $N$ for the long polymers. In contrast, $I_{P}$ shows a steep dependence on $N$ for polymers shorter than $N \approx 12$, below which $I_{P} \sim(1-\alpha N)$, where $\alpha$ is a constant. The transition point corresponds to $L_{12}=N a \approx 12 \times 4=48 \AA$ (assuming $a \approx 4 \AA[11,12])$. The $48 \AA$ value is remarkably close to the $\alpha$-HL pore length, $d \approx 52 \AA$, estimated from its structure [13]. This result and the roughly linear dependence of $I_{P}$ on $L$ for short polymers supports the notion that the blockade current level is proportional to the fractional volume of the pore occupied by the polymer. The transition between length-dependent and lengthindependent $I_{P}$ values at $L \approx 50 \AA$ was found in all experiments performed at different temperatures and with a variety of other polymer types.

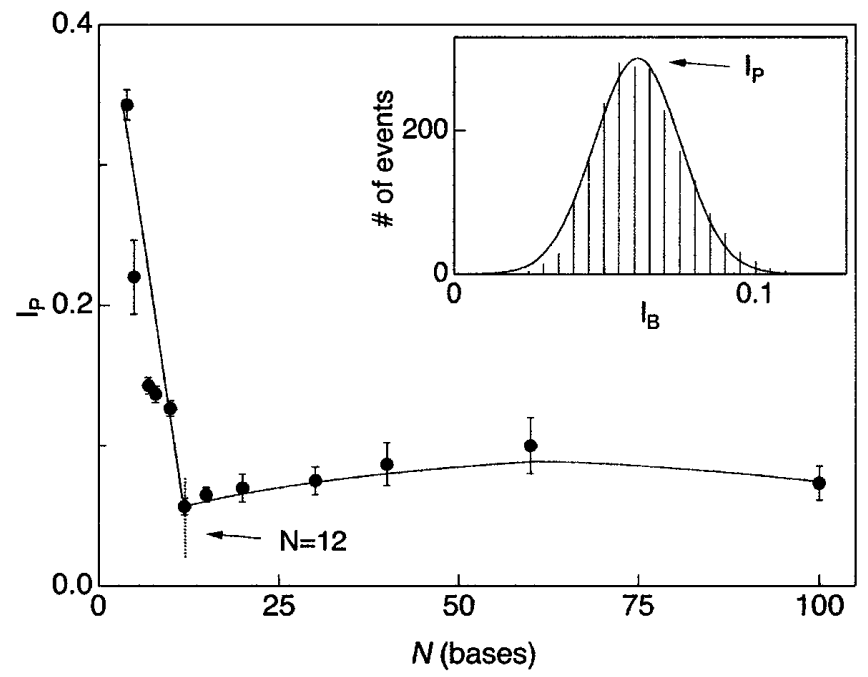

FIG. 2. The most probable blockade level, $I_{P}$, as a function of $N$ for poly(dA). $I_{P}$ depends weakly on $N$ for long polymers $(N>12)$. In contrast, $I_{P}$ has a steep dependence on $N$ for shorter polymers $(N<12)$. The transition point $(N \approx 12)$ corresponds to polymer contour length of $\sim 48 \AA$. Inset: $I_{P}$ is extracted from the translocation blockade distribution of the individual events, which is well fit by a Gaussian function. Error bars (standard error of the mean) are determined by evaluating $I_{P}$ for 3-5 data sets of the same polymer. The line is drawn to guide the eye. 
The distribution of translocation durations is more complex than a simple Gaussian [7]. For duration times shorter than the histogram peak, defined as $t_{P}$, the distribution could be well fit by a Gaussian (Fig. 3 inset). At longer times $\left(t_{D}>t_{P}\right)$, the Gaussian function does not fit our data. Instead, the distribution of $t_{D}$ values is best approximated by a falling exponential function. Consequently, $t_{P}$ differs significantly from the average duration over many translocation events, $\left\langle t_{D}\right\rangle$. The shape of the distribution resembles the simplified function derived by Lubensky et al., with a width to peak ratio of roughly 0.55 (Fig. 3 inset).

From the most probable translocation time, $t_{P}$, we calculate the apparent polymer velocities by defining $\nu_{\text {DNA }}(N)=L / t_{P}$. This velocity is independent of $N$ for long polymers $(N>12)$ with a plateau value of roughly $0.15 \AA / \mu$ s (Fig. 3, solid circles), and increases steeply for shorter polymers. Note that this velocity is roughly an order of magnitude slower than previously reported for room temperature experiments. The slow velocity is due to the strong dependence of $t_{P}$ on temperature [7]. The approximation of $\nu_{\mathrm{DNA}}=L / t_{P}$ may be improved by noticing that the polymer trajectories are somewhat larger than $L$. Because the blockade levels are proportional to the fractional volume of the pore occupied by the polymer (see above), and because $t_{P}$ is a measure of the blockade durations below $\sim 65 \%$ of $I_{\text {open }}$, we estimate the corrected trajectory during $t_{P}$ to be $L+0.35 d$. Thus, a more precise estimate of the velocity is obtained by $\nu_{\mathrm{DNA}}^{\prime}=(L+0.35 d) / t_{P}$ [14]. This velocity (solid squares, Fig. 3 ) shows a nonlinear increase for decreasing length below the threshold value

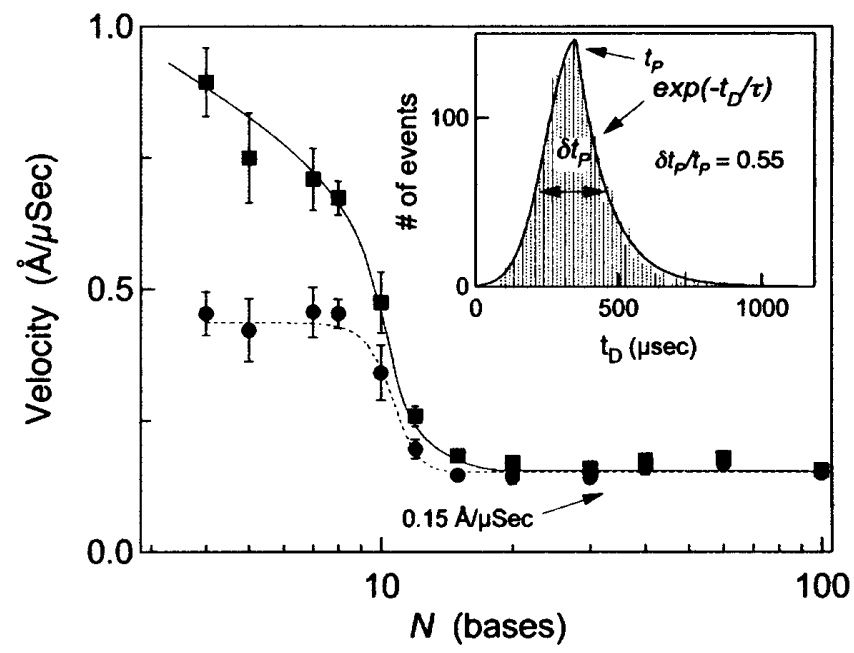

FIG. 3. Inset: A typical translocation time histogram of $\sim 1000$ events showing a non-Gaussian distribution with a peak defined as $t_{P}$ and exponential tail. The width of the distribution is defined at $t_{P} e^{-1 / 2}$. Main figure: $t_{P}$ is used to evaluate the apparent velocity $(\boldsymbol{O})$ and the corrected velocity $(\boldsymbol{\square})$, as a function of $N$. Both velocities are constant for $N>12$, with a typical plateau value of $0.15 \AA / \mu$ s at $2{ }^{\circ} \mathrm{C}$ and $120 \mathrm{mV}$. Short polymers $(N<12)$ move significantly faster through the pore than their longer counterparts. Error bars are determined as in Fig. 2. Lines are drawn to guide the eye. of $N \approx 12(L=48 \AA)$, and as expected merges with the uncorrected velocity for longer polymers. The threshold length value closely matches the length of the $\alpha$-HL pore $(d \approx 52 \AA)$, in agreement with the results presented for $I_{P}(N)$. Extrapolating our data for very short polymers, we estimate the limiting translocation velocity for a monomer of deoxyadenylic acid (at $2{ }^{\circ} \mathrm{C}$ and $120 \mathrm{mV}$ ) to be $\sim 1.4 \pm 0.2 \AA / \mu \mathrm{s}$. The plateau in the polymer velocity vs $N$ for the long polymer range is in agreement with theoretical models, which predict that $t_{P} \propto L[9,10]$. But these predictions are not valid for short polymers since $t_{P} \propto L$ was derived with the assumption of long polymers $(L \gg d)$.

To gain insight into the nonlinear behavior of the DNA polymers, we varied the electrical driving potentials from 70 to $300 \mathrm{mV}$ and measured $\nu_{\mathrm{DNA}}$ for polymers above the threshold length $(N=30$, open squares) and at the threshold length ( $N=12$, solid circles) (Fig. 4). For a restricted range of driving potentials $(70-120 \mathrm{mV})$, the translocation speed appears to depend linearly on $V$, in agreement with previous results [5]. But, over a wider range, the data no longer appears linear. A better approximation for our data over the entire range is a quadratic dependence, $\nu_{\text {DNA }}^{\prime}=k_{1}\left(V-V_{0}\right)^{2}+k_{2}$ (solid lines of Fig. 4), where $V_{0}$ is a threshold potential estimated to be $47 \pm 8 \mathrm{mV}$ based on the fit, and $k_{2}$ is a small additive constant $(\sim 0.06 \AA / \mu \mathrm{s})$. In principle, one would expect that the polymer drift velocity will approach zero at $V=V_{0}$, where the random diffusion forces dominate over the driving force. In this range, our translocation measurements may include a few polymers that randomly diffuse from one side to the other, giving rise to the small additive term, $k_{2}$.

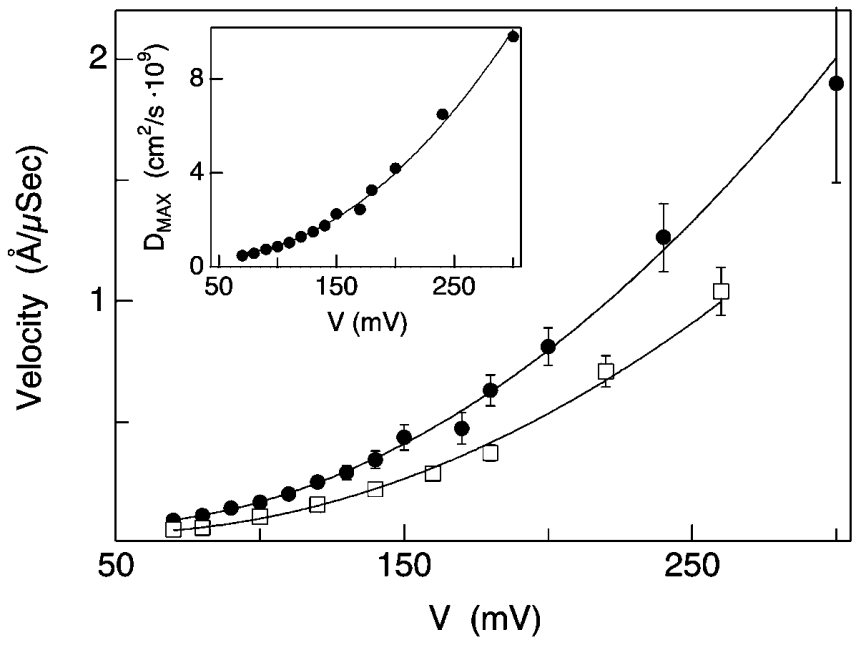

FIG. 4. The dependence of polymer velocity on the applied voltage is nonlinear. Data shown for $N=12(\mathbf{O})$ and $N=30$ ( $\square) \operatorname{poly}(\mathrm{dA}) \mathrm{s}$ at $2{ }^{\circ} \mathrm{C}$. The lines are quadratic fits to the data (see text). Error bars are determined as in Fig. 2, except for points below $90 \mathrm{mV}$ where the number of events is insufficient to determine the error bars. Inset: Estimated upper bound values for the effective diffusion coefficient, $D_{\max }$, based on the polymers' velocity. 
While it is difficult to acquire sufficient statistics near $V_{0}$ (at these low potentials only a few translocation events are observed within reasonable experimental times [14]), the finite value of $V_{0}$ may be interpreted as an energetic barrier that the system needs to overcome before translocation occurs. In the case of poly(dA) that form stacked, semiflexible polymer helices of greater diameter than the $\alpha$-HL pore $[6,7,15]$, at least 12 bases (the pore length) in the chain must be extended to a more linear structure prior to polymer sliding through the pore. This extension may involve energy penalties due to both enthalpic (unstacking) as well as entropic changes. Although a better description of polymer dynamics during pore entry will be required to assess the relative contributions of base unstacking vs entropy loss, our data provide a new estimate for the sum of these energy penalties. Evaluating the electrical energy of 12 bases extended by the field inside the pore $\left(V_{0} / d\right)$, we find $z e\left(V_{0} / d\right) a \Sigma_{n=1}^{12} n \approx 12 k_{B} T$, assuming $V_{0}=47 \mathrm{mV}$, and $z=1$. We note that $12 k_{B} T$ is an upper bounding value of the energy penalty of extending a molecule in the pore prior to translocation since in reality $z<1$. Absent a good estimate for $z$ inside the pore, we can estimate only the maximal electrical driving force as $25-110 \mathrm{pN}$ for the potential range $70-300 \mathrm{mV}$.

The relative contributions of the diffusion forces and the electrical driving force may be estimated by comparing the diffusive length (defined as $\ell_{d}=D_{\text {eff }} / \nu_{\text {DNA }}[10]$ ) to the relevant length scale $L$. When the translocation velocity approaches zero (i.e., at weak electrical potentials) $\ell_{d} \gg L$, reflecting the fact that the system is dominated by diffusion. For stronger driving potentials (i.e., for $V>120 \mathrm{mV}$ ), the steep voltage dependence that we observe suggests that in this range our system is dominated by the electrical driving force, or $\ell_{d} \ll L$. One would expect that the highly confined geometry of the DNA in the $\alpha$-HL pore would play an important role in determining the effective friction, and therefore $D_{\text {eff }}$, making it much smaller than its (free) bulk value. To estimate $D_{\text {eff }}$, a detailed microscopic model of the polymer dynamics must be undertaken. In the absence of such a model, we can set only an upper limit for $D_{\text {eff }}$ by assigning $\ell_{d} \approx L$ for the stronger potential range. The inset of Fig. 4 shows an upper bound estimation of $D_{\text {eff }}\left(D_{\text {max }}\right)$ for the confined polymer $(N=12)$ as a function of voltage. The typical values we found are in the range $(0.5-10) \times 10^{-9} \mathrm{~cm}^{2} / \mathrm{s}$. These values are more than 2 orders of magnitude smaller than the effective bulk diffusion coefficient for ssDNA having the same length [12]. We argue that these findings and the nonlinear velocity dependence on $V$ reflect the strong confinement of the DNA in the nanopore.

Our results provide quantitative estimates for the energy penalty of extending a polymer in the pore prior to translocation and the effective diffusion coefficient of short to medium length ssDNA polymers as they move through the
$\alpha$-HL pore. Although it is clear that the dynamics of short and long molecules differ considerably, a better understanding of the polymer-pore interaction and the confined geometry of the polymers in the pore will be required to explain why short molecules translocate much faster than their longer counterparts. We found that the translocation velocity is decreased by a factor of 8 for only a $7 \%$ decrease in absolute temperature [14]. This suggests that DNA-pore interactions alone cannot account for the polymer dynamics, since these interactions scale exponentially with the absolute temperature [10]. We believe that the temperature response, the strong decrease in the effective diffusion coefficient of the polymers in the pore as compared to their bulk values, and the nonlinear dependence of the velocity on the electric field are all related to the highly confined geometry of the polymers in the pore. This confinement produces a strong drag force on the translocating polymers that cannot be approximated from bulk hydrodynamics. Having set an upper bound for the energy penalty as the polymer extends into the pore and for the effective polymer diffusion coefficient, we expect that these values will form the basis for a more complete theoretical description of driven polymer translocation through a narrow pore.

We acknowledge support from the Defense Advanced Research Projects Agency (DARPA) award (No. N6523698-1-5407) and from the Rowland Institute for Science. A. M. acknowledges M. Burns, C. Cantor, J. Golovchenko, D. Lubensky, and T. Tlusty for stimulating discussions.

*Corresponding author. Electronic address: meller@rowland.org

[1] J. O. Bustamante, J. A. Hanover, and A. Liepins, J. Membr. Biol. 146, 239 (1995).

[2] B. Drieselkelmann, Microbiol. Rev. 58, 293 (1994).

[3] B. Hanss et al., Proc. Natl. Acad. Sci. U.S.A. 95, 1921 (1998).

[4] S. M. Simon and G. Blobel, Cell 65, 371 (1991).

[5] J. Kasianowicz, E. Brandin, D. Branton, and D. Deamer, Proc. Natl. Acad. Sci. U.S.A. 93, 13770 (1996).

[6] M. Akeson et al., Biophys. J. 77, 3227 (1999).

[7] A. Meller et al., Proc. Natl. Acad. Sci. U.S.A. 97, 1079 (2000).

[8] W. Sung and P. J. Park, Phys. Rev. Lett. 77, 783 (1996).

[9] M. Muthukumar, J. Chem. Phys. 111, 10371 (1999).

[10] D. K. Lubensky and D. R. Nelson, Biophys. J. 77, 1824 (1999).

[11] M. T. J. Record, C. F. Anderson, and T. M. Lohman, Q. Rev. Biophys. 11, 103 (1978).

[12] B. Tinland, A. Pluen, J. Sturm, and G. Weill, Macromolecules 30, 5763 (1997).

[13] L. Song et al., Science 274, 1859 (1996).

[14] A. Meller, L. Nivon, and D. Branton (to be published).

[15] T.G. Dewey and D.H. Turner, Biochemistry 18, 5757 (1979). 